\title{
The Role of Trypsin in The Internalization Process of Influenza H1N1 Virus into Vero and MDCK Cells
}

\author{
Fawzi Rahmadiyan Zuhairi ${ }^{1}$, Maharani ${ }^{2} \&$ Marselina I. Tan ${ }^{1}$ \\ ${ }^{1}$ Research Group of Physiology, Developmental Biology, and Biomedical Science, \\ School of Life Science and Technology, Institut Teknologi Bandung \\ Jalan Ganesa No. 10, Bandung 40132, Indonesia \\ ${ }^{2}$ Department of Research and Development, BioFarma Co, Ltd. \\ Jalan Pasteur No. 28, Bandung 40161, Indonesia \\ Email: fawzi.zuhairi@gmail.com
}

\begin{abstract}
Trypsin is supposed to play an important role in the internalization of the influenza virus into host cells in vitro because of its protease activity. The purpose of this study is to determine the role of trypsin in the internalization process of the influenza H1N1 virus in Vero and MDCK (Madin-Darby Canine Kidney) cells; to see whether trypsin can trigger the endocytosis route via receptor-mediated endocytosis or non-receptor-mediated endocytosis. The viruses were inoculated into Vero and MDCK cells under three conditions: (1) without trypsin in the medium (VVi and MVi); (2) with medium containing trypsin (VTVi1 and MTVi1); and (3) cells washed with trypsin prior to virus inoculation and incubated with medium containing trypsin (VTVi2 and MTVi2). Advance cytopathic effects (CPE) could only be observed in the MDCK cells. HA (hemagglutination) titer on the MDCK cells was higher than that on the Vero cells. Moreover, HA titer on MTVil was higher than MVi, and the lowest was found on MTVi2. Trypsin supported the internalization of the influenza virus by the cleavage of HA glycoprotein into HA1 and HA2. However, trypsin could halt the internalization of the influenza virus when the MDCK cells were washed with trypsin before virus inoculation. Trypsin could digest transmembrane protein containing sialic acid that is important for the virus internalization. Conversely, trypsin did not affect the internalization of the influenza virus in the Vero cells based on HA titer. This result was also supported by TEM observation, which showed that the ability of Vero cellsto internalize the influenza virus is very low. We suggest that a limited amount of sialic acid $(\alpha-$ 2,6)-containing receptor on the membrane of the Vero cells can halt influenza virus internalization. On the other hand, active internalization of the virus was seen in all groups of MDCK cells (MVi, MTVi1, and MTVi2). However, trypsin could not trigger the internalization of the influenza virus in Vero and MDCK cells via non-receptor-mediated endocytosis. From this study it can be concluded that trypsin enhanced the internalization of influenza virus into MDCK cells by cleavage of the hemagglutinin (HA) of the virus, but did not improve the ability of the host cells to internalize the virus, especially via non-receptor-mediated endocytosis.
\end{abstract}

Keywords: influenza; MDCK cell; trypsin; vero cell.

Copyright ( 2012 Published by LPPM ITB, ISSN: 1978-3043, DOI: 10.5614/itbj.sci.2012.44.4.1 


\section{$1 \quad$ Introduction}

Influenza is a disease that is caused by influenza viruses, which can cause annual epidemics and global pandemic diseases in humans. The emergence of new strains will continue to harm humans and other mammalians and also challenge public health and scientific communities [1]. Vaccination is one of the best ways to prevent diseases, such as influenza [2].

Embryonated chicken eggs are used routinely for human influenza virus vaccines production. However, this cultivation method lead to the selection of variants characterized by antigenic and structural change in the hemagglutinin (HA) molecule [3-5]. Moreover, there are some other disadvantages of using embryonated chicken eggs, such as the uncertainty of a reliable year-round supply of high-quality eggs and also the presence of adventitious agents $[6,7]$.

Influenza viruses are able to replicate in a variety of primary, diploid, and continuous cell cultures [8]. Influenza viruses grow optimally in MDCK (Madin-Darby Canine Kidney) cells. However, this cell line has not been licensed as a substrate for vaccine production because it has a tumorigenic character $[7,9]$.

The African green monkey kidney (Vero) cell line is fully characterized and approved by the World Health Organization (WHO) for the production of human vaccines against poliomyelitis and rabies [10]. This cell line has the potential to be developed as an alternative cell-based system for influenza vaccine production. However, some research has shown that not all influenza virus strains grow optimally in Vero cell [11]. Treatment with trypsin of the culture medium can improve the virus yield [12].

Trypsin is supposed to be essential for the improvement of the infectivity of the influenza virus on mammalian cell lines. As a protease, it cleaves the influenza hemagglutinin (HA) component into two sub-units, HA1 and HA2, that are held together by a disulfide link [12]. Virions containing uncleaved HA are noninfectious [13]. Trypsin is also routinely used for enzymatic disaggregation of cell cultures. As a protease, trypsin can digest many protein membranes that have a function in cell-cell adhesion or cell-substrate adhesion [14].

The internalization of the influenza virus occurs in four ways: micropinocytosis, clathrin-mediated endocytosis, caveolin-mediated endocytosis, and nonclathrin- and non-caveolin-mediated endocytosis [10]. Rust, et al. [15] found that non-clathrin- and non-caveolin-mediated endocytosis were involved in the internalization of influenza viruses with a percentage of up to $35 \%$, whereas clathrin- and caveolin-mediated endocytosis contributed to $65 \%$ and 5\% 
respectively in the internalization process of influenza viruses. Based on the protease activity of trypsin, we have suggest that the internalization of influenza viruses, especially via non-receptor-mediated endocytosis or micropinocytosis, may be enhanced by trypsin. Therefore, we would like to know the role of trypsin in the internalization process of the influenza virus into Vero and MDCK cells. This study is to present a comparison of the influenza virus H1N1 A/Solomon Island/3/06-like internalization in Vero and MDCK cell lines treated with trypsin.

\section{$2 \quad$ Materials and Methods}

\subsection{Cultivation of Vero and MDCK Cell Line}

The Vero cell line was obtained from the American Type Culture Collection (Rockville, MD). The cells were cultivated at $36,5{ }^{\circ} \mathrm{C}$ with basal medium, consisting of Dulbecco's Modification of Eagle's Medium (DMEM), with 0,1\% sodium bicarbonate $\left(\mathrm{NaHCO}_{3}\right), 15 \mu \mathrm{g} / \mathrm{ml}$ of erythromycin, and $75 \mu \mathrm{g} / \mathrm{ml}$ kanamycin. The cells were also supplemented with $10 \%$ Bovine Calf Serum (BCS). Every 7 days, vero cells were subcultured.

The MDCK cells were kindly provided by PT. Bio Farma, Bandung, Indonesia. The cells were maintained at $36,5^{\circ} \mathrm{C}$ with basal medium, consisting of Eagle minimal essential medium (MEM), with $0,1 \%$ of sodium bicarbonate $\left(\mathrm{NaHCO}_{3}\right), 15 \mu \mathrm{g} / \mathrm{ml}$ erythromycin, and $75 \mu \mathrm{g} / \mathrm{ml}$ kanamycin (EK). The MDCK cells were also supplemented with $10 \%$ Bovine Calf Serum (BCS). The MDCK cells were subcultured every 4 days.

\subsection{Influenza Virus Propagation}

The influenza virus strain H1N1 A/Solomon Island/3/06-Like was obtained from PT. Bio Farma (Bandung, Indonesia) and propagated in 11-old-day embryonated chicken eggs. The viruses were harvested three days after inoculation and stored at $-80^{\circ} \mathrm{C}$ until used.

\subsection{Influenza Virus Inoculation on Vero and MDCK Cell Line and Haemagglutination Test}

The Vero and MDCK cells were seeded each into twenty $25-\mathrm{cm}^{2}$ flasks, and allowed to reach a subconfluent condition in two days for virus inoculation. In the MK, VK, MT, and VT groups, cells were washed with phosphate buffer saline (PBS) twice and layered with korioalantoic fluid for 1 hour at $34{ }^{\circ} \mathrm{C}$. The flasks were then added with basal medium for the MK and VK groups, and with basal medium containing $0,5 \mu \mathrm{g} / \mathrm{ml}$ trypsin TPCK (L-l-tosylamide-2- 
phenylethyl chloromethyl ketone-treated, Sigma, Cat. T1426) for the MT and VT groups.

For the MVi and VVi groups, the cells were washed with PBS twice and inoculated with $2560 \mathrm{HA}$ units of influenza virus for 1 hour at $34{ }^{\circ} \mathrm{C}$, and then added with basal medium. For the MTVil and VTVil groups, the cells were washed with PBS and then inoculated with the influenza virus for 1 hour at 34 ${ }^{\circ} \mathrm{C}$, and then were added with basal medium containing $0,5 \mu \mathrm{g} / \mathrm{ml}$ trypsin. For the MTVi2 or VTVi2 groups, the cells were washed with $0,5 \mu \mathrm{g} / \mathrm{ml}$ trypsin and then inoculated with the influenza virus for 1 hour at $34{ }^{\circ} \mathrm{C}$ and added with basal medium containing $0,5 \mu \mathrm{g} / \mathrm{ml}$ trypsin.

The cell cultures were observed every 24 hours for six days to detect the presence of cytopathic effect (CPE), and $0,5 \mathrm{ml}$ of supernatan was taken and stored at $-80{ }^{\circ} \mathrm{C}$ until it was used for the HA test.

\subsection{TEM Observation}

The MDCK and Vero cells were grown on aclar film (purchased from Electron Microscopy Sciences, \#50426-25, $51 \mu \mathrm{m}$ thick) and were supplemented with basal medium containing 10\% bovine calf serum (BCS). After 24 hours, the cell cultures were inoculated with 1843,2 HA units of influenza virus. The infected monolayer cells were fixed at 48 hours post infection in cacodylate-buffered $2,5 \%$ glutaraldehyde, postfixed in $2 \%$ osmium tetroxide, dehydrated in a series of ethanols, and embedded in Spurr embedding medium. Ultrathin sections of cells were further stained with uranyl salt and tryple lead, and observed using a JEOL Electron Microscope, operated at $80 \mathrm{kV}$.

\section{$3 \quad$ Results and Discussion}

Advanced CPE (Cytopathic effects, Figure 1(a)) were found in the MDCK cells that were inoculated with the influenza virus, especially in the group containing trypsin (MTVi1 group), while the least advanced CPE was found in the group that was treated with trypsin before virus inoculation (MTVi2). In addition, detachment of all cells the MVi, MTVi1, and MTVi2 groups was found from the third day after inoculation in the MVi and MTVil cells, and from the fifth day after inoculation in the MTVi2 cells (data not shown). Based on this result, the influenza virus was internalized very well in the MDCK cells, and the virus that had been treated with trypsin enhanced the internalization of the influenza virus into the MDCK cells. However, the MDCK cells treated with trypsin before virus inoculation halted the internalization of the influenza virus into the MDCK cells. The occurence of CPE in the infected cell monolayer is one of the evidences of viral growth [16]. CPE that are caused by an influenza virus have 
several characteristics, such as the formation of enlarged focal vacuolation of the cells, cell deterioration, followed in advanced CPE condition, by detachment of the cells from the substrate [17].

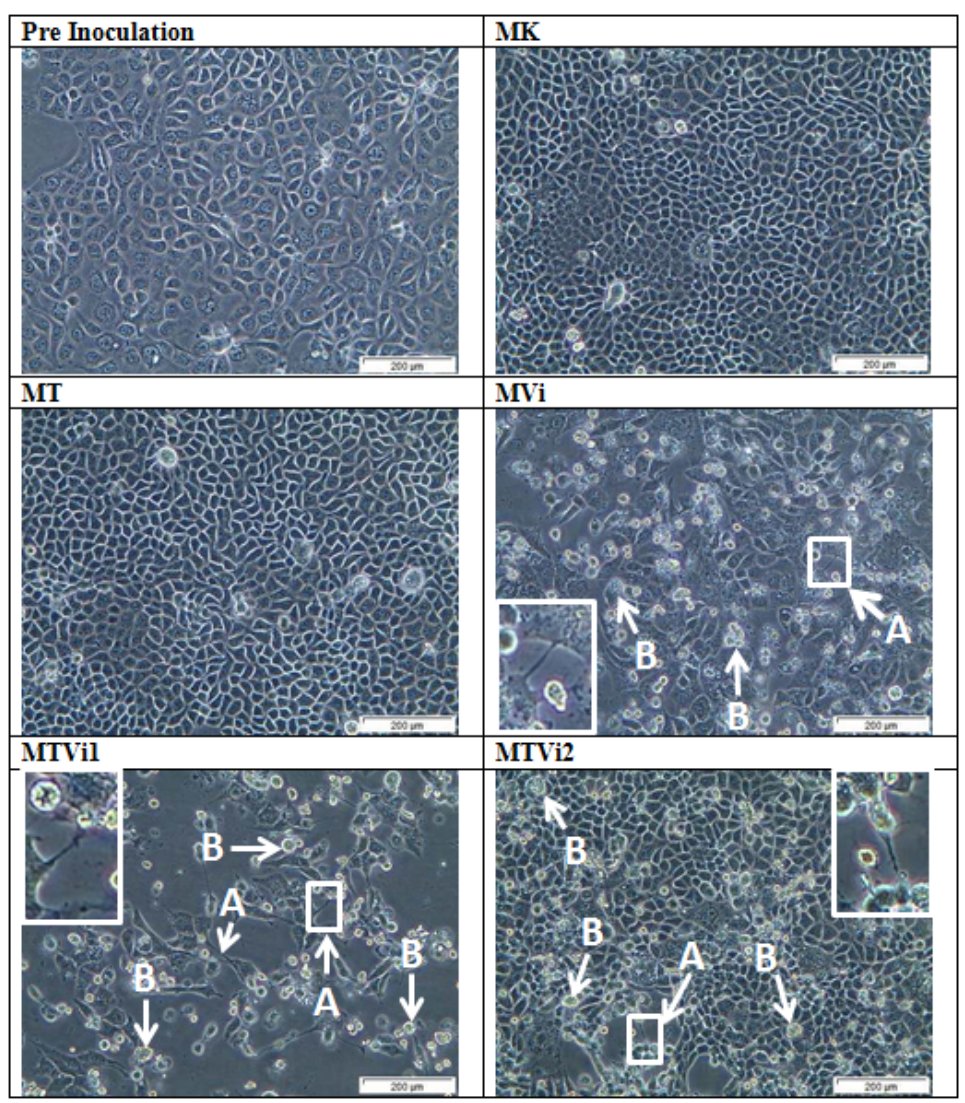

Figure 1 (a) Observation of MDCK cells using inverted microscope. Note: PreInoculation: MDCK cells that were grown with complete medium before virus inoculation. MK: MDCK cells that were grown with basal medium, MT: MDCK cells that were grown with basal medium containing trypsin $0,5 \mu \mathrm{g} / \mathrm{ml}, \mathrm{MVi}$ : MDCK cells that were inoculated with influenza virus and grown with basal medium, MTVi1: MDCK cells that were inoculated with influenza virus and grown with basal medium containing trypsin $0,5 \mu \mathrm{g} / \mathrm{ml}$, MTVi2: MDCK cells that were washed with trypsin $0,5 \mu \mathrm{g} / \mathrm{ml}$ prior to virus inoculation and grown with basal medium containing trypsin $0,5 \mu \mathrm{g} / \mathrm{ml}$. MK, MT, MVi, MTVi1, and MTVi2 were observed two days after virus inoculation. A\&B: cytopathic effect characteristic. A: cytoplasmic bridge structure (with inset box), B: rounding of the cells. 


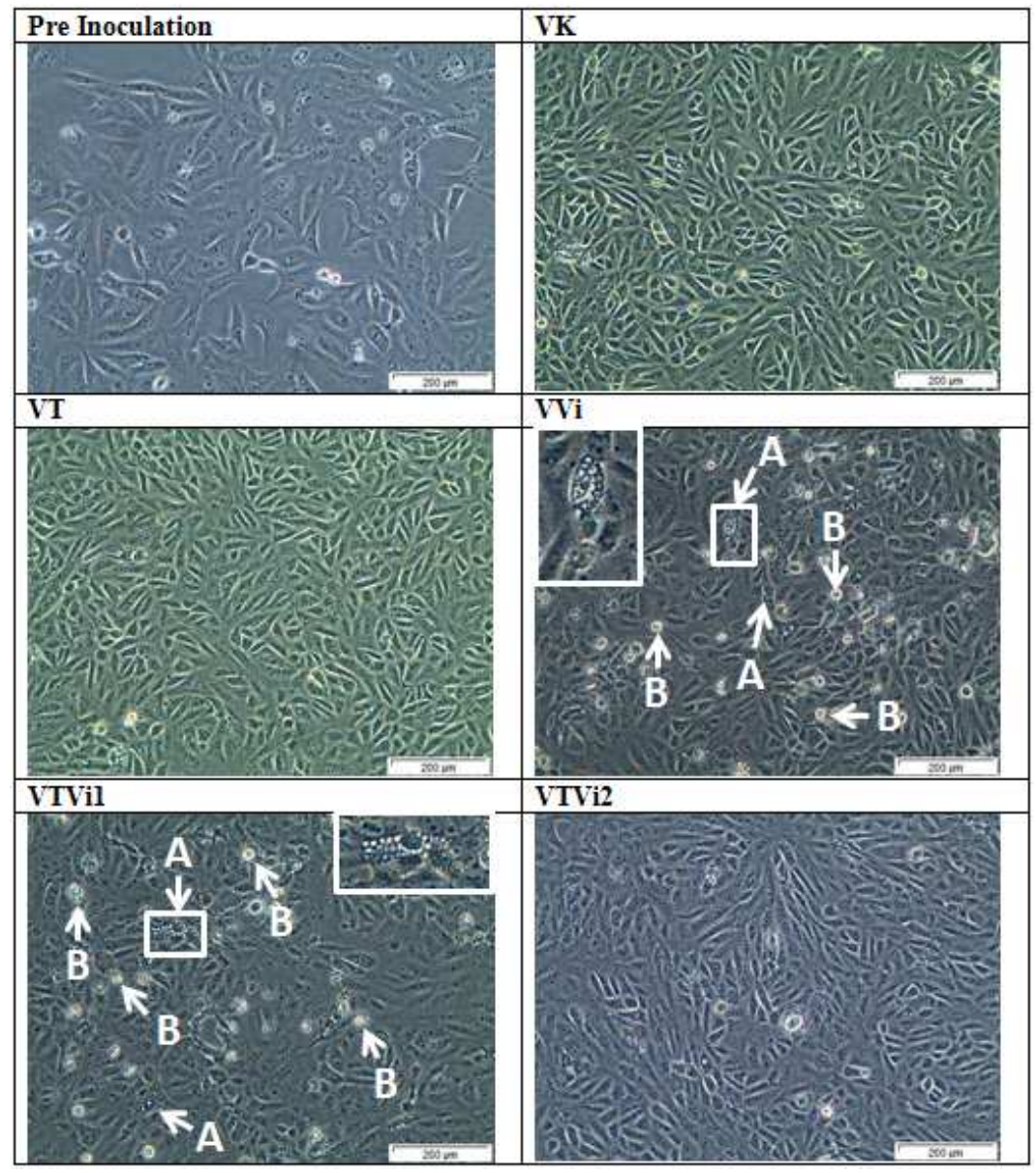

Figure 1 Continued. (b) Observation of vero cells using inverted microscope. Note: Pre-Inoculation: vero cells that were grown with complete medium before virus inoculation. VK: Vero cells that were grown with basal medium, VT: Vero cells that were grown with basal medium containing trypsin $0,5 \mu \mathrm{g} / \mathrm{ml}, \mathrm{VVi}$ : Vero cells that were inoculated with influenza virus and grown with basal medium, VTVi1: Vero cells that were inoculated with influenza virus and grown with basal medium containing trypsin $0,5 \mu \mathrm{g} / \mathrm{ml}$, VTVi2: Vero cells that were washed with trypsin $0,5 \mu \mathrm{g} / \mathrm{ml}$ prior to virus inoculation and grown with basal medium containing trypsin $0,5 \mu \mathrm{g} / \mathrm{ml}$. VK, VT, VVi, VTVi1, and VTVi2 were observed two days after virus inoculation. A\&B: cytopathic effect characteristic. A: vacuolation of the cell (with inset box), B: rounding of the cells. 


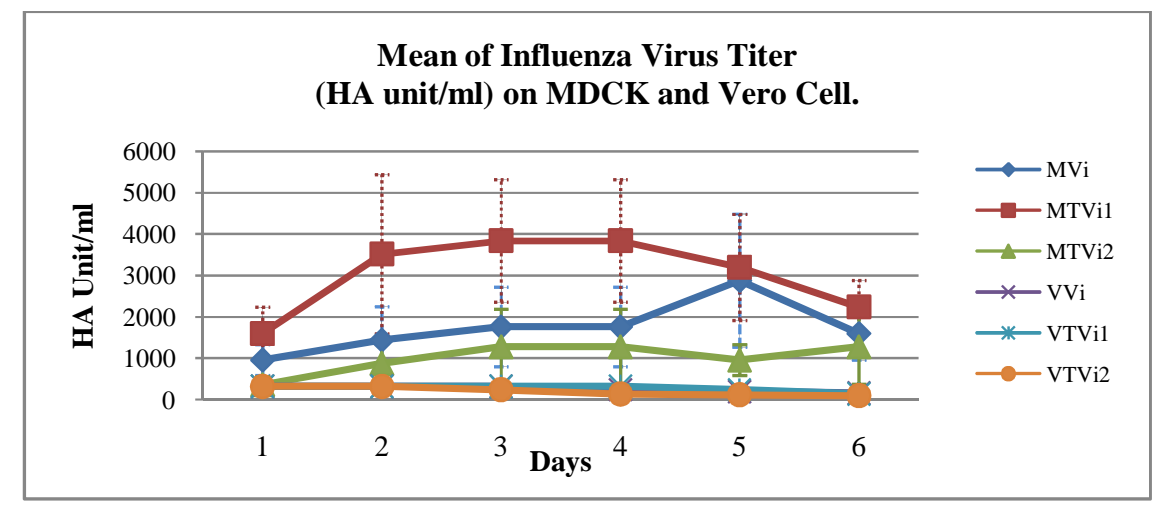

Figure 2 Graphic of Influenza Virus HA (Unit/ml) Titer in MDCK and Vero Cells.

Table 1 Mean titer of HA Unit/ml with standard deviation (HA unit/ml \pm Standard Deviation). MK \& VK: MDCK \& Vero cells that were grown with basal medium, MT \& VT: MDCK \& vero cells that were grown with basal medium containing trypsin $0,5 \mu \mathrm{g} / \mathrm{ml}$, MVi\&VVi: MDCK \& vero cells that were inoculated with influenza virus and grown with basal medium, MTVi1\& VTVi1: MDCK \& vero cells that were inoculated with influenza virus and grown with basal medium containing trypsin $0,5 \mu \mathrm{g} / \mathrm{ml}$, MTVi2\& VTVi2: MDCK \& vero cells that were washed with trypsin $0,5 \mu \mathrm{g} / \mathrm{ml}$ prior to virus inoculation and grown with basal medium containing trypsin $0,5 \mu \mathrm{g} / \mathrm{ml}$.

\begin{tabular}{ccccccc}
\hline \multirow{2}{*}{ Group } & \multicolumn{7}{c}{ Days } \\
\cline { 2 - 7 } & $\mathbf{1}$ & $\mathbf{2}$ & $\mathbf{3}$ & $\mathbf{4}$ & $\mathbf{5}$ & $\mathbf{6}$ \\
\hline MVi & $960 \pm 369$ & $1440 \pm 805$ & $1760 \pm 960$ & $1760 \pm 960$ & $2880 \pm 1610$ & $1600 \pm 640$ \\
\hline MTVi1 & $1600 \pm 640$ & $3520 \pm 1920$ & $3840 \pm 1478$ & $3840 \pm 1478$ & $3200 \pm 1280$ & $2240 \pm 640$ \\
\hline MTVi2 & $360 \pm 201$ & $880 \pm 480$ & $1280 \pm 905$ & $1280 \pm 905$ & $960 \pm 369$ & $1280 \pm 905$ \\
\hline VVi & $320 \pm 0$ & $320 \pm 0$ & $320 \pm 0$ & $280 \pm 92$ & $240 \pm 80$ & $160 \pm 0$ \\
\hline VTVi1 & $320 \pm 0$ & $320 \pm 0$ & $320 \pm 0$ & $320 \pm 0$ & $320 \pm 92$ & $220 \pm 40$ \\
\hline VTVi2 & $320 \pm 0$ & $320 \pm 0$ & $240 \pm 92$ & $180 \pm 40$ & $160 \pm 46$ & $100 \pm 40$ \\
\hline
\end{tabular}

Based on the HA titer test (Figure 2 and Table 1), the influenza virus was internalized very well in thr MDCK cells. The Mann-Whitney $U(\alpha=0,05)$ test showed that the HA titer was significantly different $(\mathrm{p}<0,05)$ between the MVi and VVi, MTVi1 and VTVi1, and MTVi2 and VTVi2 groups. A previous study [7] has shown that the distribution of this receptor is high in MDCK cells. The MDCK cells that were treated with trypsin (MTVi1) showed the highest HA titer, while in the MTVi2 group, the MDCK cells that were treated with trypsin before virus inoculation had the lowest HA titer. Based on this result, trypsin 
can enhance the internalization and replication of the influenza virus only in MDCK cells. The cleavage of the virus HA (hemagglutinin) into HA1 and HA2 enhances the infectivity of the virus by inducing the fusion of the virus with the endosomal membrane, so the virus can transfer the genetic material into the nucleus $[18,19]$. However, we suggest that trypsin treatment prior to virus inoculation obstruct the internalization of virus by the impairment of receptor protein SA ( $22-6)$ Gal, which is essential for the endocytosis of influenza virus, because of the protease activity of trypsin can degrade this receptor protein.

In the Vero cells from the VTVil group, which were treated with trypsin, and the VVi group, there were only few cells that showed CPE (Figure 1(b)). Moreover, Vero cells from every group could grow well until they were confluent on the 6th day, even when the viruses were cultivated with trypsin (data not shown). This result shows that the internalization of the infleunza virus in Vero cells was not as well as in the MDCK cells, which was also supported by the HA titer test (Figure 2 and Table 1). We suggest that the lack of distribution of the receptor protein containing sialic acid ( $\alpha 2-6)$ or SA ( $\alpha 2-6)$ Gal on the Vero cells halts the internalization of the influenza virus [7]. Receptor protein SA ( $\alpha 2-6)$ Gal is improtant in the early step of the virus internalization process because virus will not be internalized by the cell unless the virus attaches to the SA ( $\alpha 2-6) \mathrm{Gal}$, thus trigger the endocytosis of the virus into the cell [19]. Therefore, the lesser the amount of SA ( $\alpha 2-6) \mathrm{Gal}$, the lesser the internalization of the virus. The internalization of the influenza virus into the Vero cells could also be impaired by the low infectivity of influenza virus progeny as a result from abortive replication of the virus in the Vero cells [11].

Minimum internalization of the influenza virus into the Vero cells was supported by TEM observation in our study, which showed that only few viruses could noticeably be internalized by the Vero cells (Figure 3). This shows that trypsin could not enhance the Vero cells' ability to internalize the influenza virus. This result also shown that the availability of receptor protein SA ( $\alpha 2-6)$ Gal was still the major factor for the internalization of the influenza virus into host cell.

In contrast to the Vero cells, the virus could be internalized by the MDCK cells actively. We suggest that the internalization of the influenza virus into the MDCK cells is mediated by clathrin protein (Figure 3) based on the visible coat in the membrane where the internalization of the virus occurs. The clathrinmediated internalization is also supported by previous studies from Matlin, et al. [20] and Rust, et al. [15]. TEM observation results show that the treatment of trypsin did not affect the ability of the MDCK cell membrane, and also vero cell membrane, to internalize the virus. In addition, trypsin also did not affect the route of influenza internalization into the MDCK and vero cells since there 
is no evidence of the non-receptor mediated endocytosis or micropinocytosis of the influenza virus into the cells.

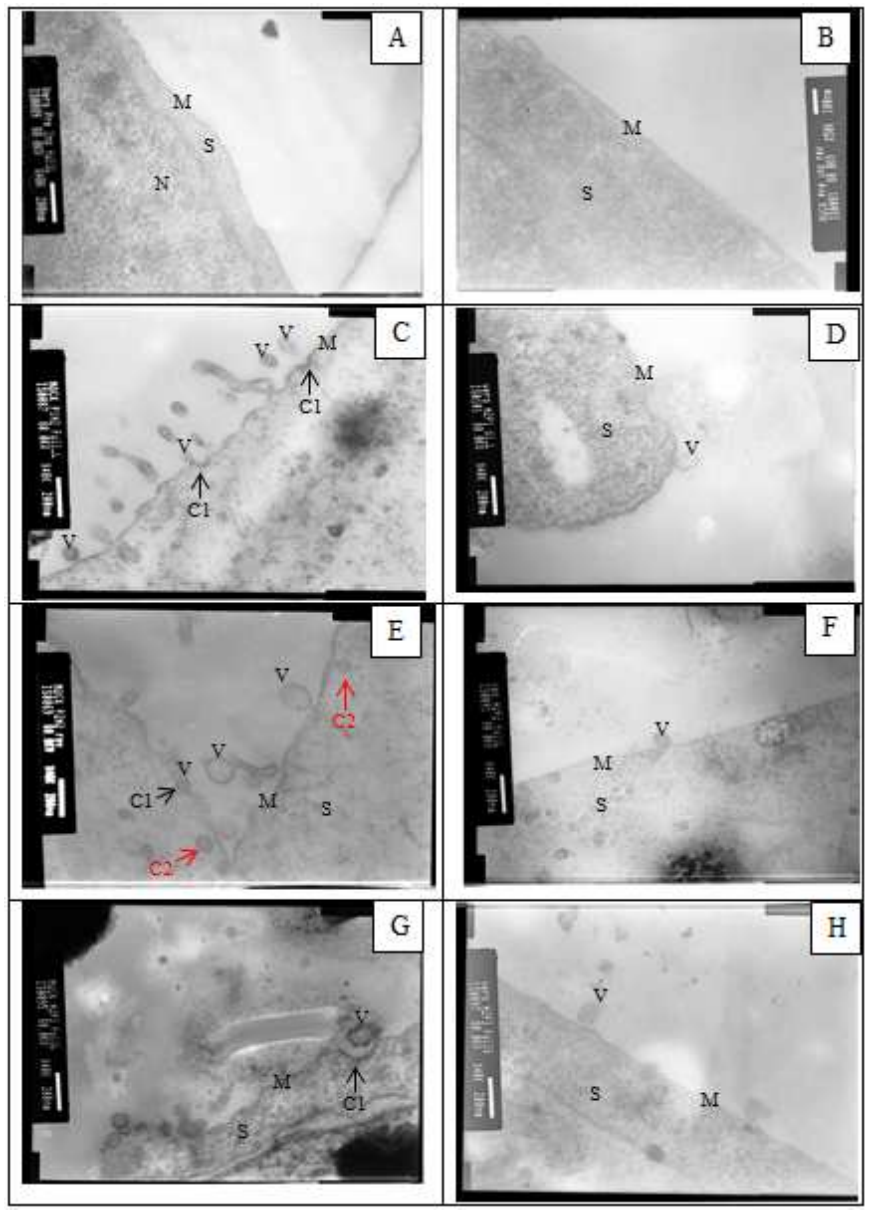

Figure 3 Observation of the internalization process of influenza virus into vero and MDCK cells using Transimission Electron Microscope (TEM). A \& B : PreInoculation condition of MDCK (A) and vero cell (B). C- H: observed in 48 hours after inoculation. C \& D: MVi \& VVi, E \& F: MTVi1 \& VTVi1, G \& H: MTVi2 \& VTVi2. M: Cell membrane, N: Nucleus, S: Cytoplasm, C1: Clathrincoated pit, C2: Clathrin-coated vesicle. Bar: $200 \mathrm{~nm}$.

\section{Conclusion}

Based on the CPE and HA titer test results, trypsin facilitated a better internalization of the influenza virus into the MDCK cells, whereas the 
internalization of the virus into the Vero cells was limited due to the minimum distribution of the receptor protein SA $(\alpha 2,6) \mathrm{Gal}$. We have suggested that the presence of the receptor protein SA $(\alpha 2,6) \mathrm{Gal}$ in the host cell is more important than the infectivity of the virus triggered by the cleavage of the HA of the virus by tripsin.

The CPE observation and HA titer results are supported by observation of the internalization process using TEM, which showed limited interaction between the influenza virus and the Vero cell, and conversely, an obvious binding and internalization process of the virus in the MDCK cells. However, trypsin did not affect the ability of the MDCK and Vero cells to internalize the influenza virus, especially via non-receptor-mediated endocytosis or micropinocytosis. Based on this study, it can be concluded that trypsin can only affect the cleavage of influenza hemagglutinin to enhance the internalization of the influenza virus by the host cells.

\section{Acknowledgements}

This study was funded by Bio Farma, Bandung, Indonesia.

\section{References}

[1] Neumann, G., Noda, T., \& Kawaoka, Y., Emergence and Pandemic Potential of Swine-Origin HIN1 Influenza Virus, Nature, 459(7249), pp. 931-939, 2009.

[2] Kindt, T.J., Goldsby, R.A., Osborne, B.A. \& Kuby, J., Kuby Immunology, $6^{\text {th }}$ edition, W.H. Freeman, ed., pp. 413, 2007.

[3] Katz, J.M., Naeve, C.W. \& Webster, R.G., Host Cell Mediated Variation in H3N2 Influenza Viruses, Virology, 156(2), pp. 386-395, 1987.

[4] Robertson, J.S., Naeve, C.W., Webster, R.G., Bootman, J.S., Newman, R. \& Schild, G.C., Alterations in the Hemagglutinin Associated with Adaptation of Influenza B virus to Growth in Eggs, Virology, 143(1), pp. 166-174, 1985.

[5] Schild, G.C., Oxford, J.S., de Jong, J.C.\& Webster, R.G., Evidence for Host-Cell Selection of Influenza Virus Antigenic Variants, Nature, 303(5919), pp. 706-709, 1983.

[6] Monto, A.S., Maassab, H.F. \& Bryan, E.R., Relative Efficacy of Embryonated Eggs and Cell Culture for Isolation of Contemporary Influenza Viruses, J. Clin. Microbiol., 13(1), pp. 233-235, 1981.

[7] Govorkova, E.A., Murti, G., Meigner, B., Taisne, D.\& Webster, R.G, African Green Monkey Kidney (Vero) Cells Provide anAlternative Host Cell System for Influenza A and B Viruses, Journal of Virology, 70(8), pp. 5519-5524, 1996. 
[8] Kilbourne, E.D., Cytopathogenesis and Cyto-pathology of Influenza Virus Infection of Cells in Culture, Influenza, In E.D. Kilbourne, ed., Plenum Publishing Corp., New York, 1987.

[9] Ozaki, H., Govorkova, E.A., Li, C., Xiong, X., Webster, R.G.\& Webby, R.J., Generation of High-Yielding Influenza A Viruses in African Green Monkey Kidney (Vero) Cells by Reverse Genetics, Journal of Virology, 78(4), pp. 1851-1857, 2004.

[10] Montagnon, B.J., Fanget, B.\& Nicolas, A.J., The Large Scale Cultivation of Vero Cells in Micro-Carrier Culture for Virus Vaccine ProductionPreliminary Results for Killed Poliovirus Vaccine, Developments in Biological Standardization, 47, pp. 55-64, 1981.

[11] Lau, C.L. \& Scholtissek, C., Abortive Infection of Vero Cells by an Influenza A Virus (FPV), Virology, 212(1), pp. 225-231, 1995.

[12] Kaverin, N.A. \& Webster, R.G.,Impairment of Multicycle Influenza Virus Growth in Vero (WHO) Cells by Loss of Trypsin Activity, Journal of Virology, 69(4), pp. 2700-2703, 1995.

[13] Webster, R.G. \& Rott, R., Influenza Virus A Pathogenicity: The Pivotal Role of Hemagglutinin, Cell, 50(5), pp. 656-666, 1987.

[14] Freshney, R.I., Culture of Animal Cells, $5^{\text {th }}$ edition, John Willey and Sons, pp. 32, 2006.

[15] Rust, M.J., Lakadamyali, M., Zhang, F. \& Zhuang, X, Assembly of Endocytic Machinery Around Individual Influenza Viruses During Viral Entry, Nat. Struct. Mol. Biol., 11(6), pp. 567-573, 2004.

[16] George, V.G., Hierholzer, J.C. \& Ades, E.W., Cell Culture, In Mahy, Brian, W.J.\& Kangro, H.O., eds., Virology Methods Manual, London: Academic Press, 1996.

[17] Hamilton, S.B., Wyatt, D.E., Wahlgren, B.T., O’ Dowd, M.K., Morissey, J.M., Daniels, D.E. \& Lednicky, J.A., Higher Titers of Some H5N1 and Recent Human H1N1 and H3N2 Influenza Viruses in Mv1 vs MDCK Cells, Virology Journal, 8, pp. 66-70, 2011.

[18] Shaw, M.L. \& Palese, P., Orthomyxoviruses: Molecular Biology, In: Mahy, B.W.J. \& Regenmortel, M.H.V.V., eds., Encyclopedia of Virology, $3^{\text {rd }}$ edition, pp. 483-489, Slovenia: Elsevier,2008.

[19] Zambon, M. \& Potter, C.W., Influenza, In: Zuckerman, A.J., Banatvala, J.E., Schoub, B.D., Griffiths, P.D. \& Mortimer, P., eds., Principles and Practices of Clinical Virology, $6^{\text {th }}$ edition, pp. 373-408, Singapore: John Willey and Sons, 2009.

[20] Matlin, K.S., Reggio, H., Helenius, A. \& Simons, K., Infectious Entry Pathway of Influenza-Virus In A Canine Kidney-Cell Line, J. Cell Biol, 91(3), pp. 601-613, 1981. 